\title{
CORRESPONDENCE
}

\section{NEUROCYSTICERCOSIS AND AFEBRILE SEIZURE}

Sir,

The report on "neurocysticercosis and afebrile seizure" "is very interesting. SAHU et al. noted that "neurocysticercosis should be suspected in every child presenting with afebrile seizure especially with a radio-imaging supportive diagnosis in tropical developing countries or areas endemic for teniasis/cysticercosis 3 ". In fact, neurocysticercosis is a very important tropical neurological infection. This disease can be seen worldwide. The MRI finding and immune response in neurocysticercosis is very interesting. KISHORE et al. found that "immune response was sub-optimal even in MRI positive cases and conversely, few MRI negative cases were seropositive ${ }^{1}$." Hence, to diagnose, both investigations are necessary ${ }^{1}$. Furthermore, there are many concerns on treatment of the neurocysticercosis presenting with afebrile seizure. First, the efficacy of antiparasitic drug might be reduced due to drug interaction with antiepileptic drug ${ }^{2}$. To manage the case of neurocysticercosis, searching for possible infestation in other organs of the patient is suggested since disseminated infestation can be expected. Also, other concomitant parasitic infestation must be searched for.

Viroj WIWANITKIT

Visiting professor, Hainan Medical University, China; visiting professor, Faculty of Medicine, University of Nis, Serbia; adjunct professor, Joseph Ayobabalola University, Nigeria; professor, senior expert, Surin Rajabhat, Thailand.

Correspondence to: Professor Viroj Wiwanitkit Wiwanitkit House, Bangkhae, Bangkok Thailand 10160.

E-mail: wviroj@yahoo.com

\section{REFERENCES}

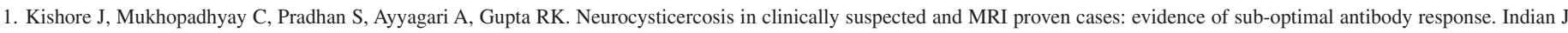
Pathol Microbiol. 2004;47:290-4.

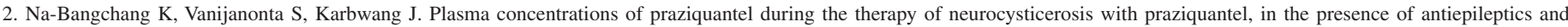
dexamethasone. Southeast Asian J Trop Med Public Health. 1995;26:120-3.

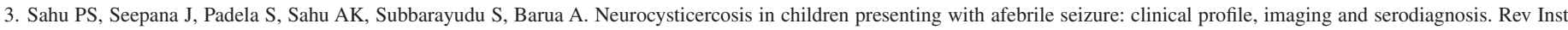
Med Trop Sao Paulo. 2014;56:253-8. 